\title{
Fully natural garment dyeing using tamarind leaves as mordant
}

\begin{abstract}
In spite the use of synthetic dyes, natural dyes are still subjugated and used to enhance its inherent aesthetic qualities as a major material for embellishment of the body. Centuries before the discovery of synthetic dyes, natural dyes were the only source of dye open to mankind. Dyes are extracted from plant -leaves, roots and barks, insect secretions, and minerals. However, research findings have made it clear that of all, plants- leaves, roots, barks or flowers are the most explored and exploited. During the normal processes, different types of chemicals are used for functional purpose \& aesthetic issues. All the chemicals are produced through a series of reaction \& have the minimal amount of side effects to our environment \& human ecology. Without the use of dyes \& chemicals, normally this is undesirable to get colored product. Specially for Natural dyeing, chemical mordants are used though the dye is natural. This paper explores dyeing with natural dye \& tamarind leaves as natural mordant. And performance of natural mordant was compared to chemical mordant as well.
\end{abstract}

Keywords: turmeric, tamarind leaves, natural mordant, feso $_{4}$
Volume 3 Issue 3 - 2017

\author{
Shaikh Mohammad Mominul Alam,' Salma \\ Katun Sela, ${ }^{2}$ AKM Nayab-Ul-Hossain, ${ }^{3}$ \\ Mohammad Masud Kaisar Sajib, ${ }^{4}$ Mohammad \\ Rakibul Hasan ${ }^{5}$ \\ 'Faculty of Science \& Engineering, Bangladesh University of \\ Textiles, Dhaka, Bangladesh \\ ${ }^{2}$ Woven Dyeing Laboratory, Thermax Woven Dyeing Limited, \\ Narsingdi, Dhaka, Bangladesh \\ ${ }^{3,4.5}$ Department of Textile Engineering, Khulna University of \\ Engineering \& Technology, Khulna, Bangladesh
}

Correspondence: Shaikh Mohammad Mominul Alam, Faculty of Science \& Engineering, Bangladesh University of Textiles, Dhaka, Bangladesh, Email dalim70@yahoo.com

Received: November 06, 2017 | Published: November 24, 2017

\section{Introduction}

The use of dye stuffs is as old as textiles themselves and pre-dates written history. Dyes are obtained from two main sources; the natural dyes and synthetic dyes. ${ }^{1}$ Natural dyes can be defined as those organic materials that have the ability to impart colour to any substrates which they must have had affinity for. ${ }^{2}$ Natural dyes are biodegradable and very compatible with the environment. They have beauty and depth of colour that cannot quite be obtained with synthetics. These dyes can be obtained either from plants, animals, and minerals. ${ }^{3}$ Until the mid 19th century, all dyestuffs were made from natural materials, mainly vegetables matter. ${ }^{2}$ Research findings have reported that synthetic dyes are harmful to the body and thus the increased search into the arrays of plants for natural dyes which is more environmental friendly. ${ }^{4}$ Most of these dyes are substantive and mordant are used to increase the colour and quality fabric. In this article work, no textile chemicals, auxiliaries \& textile dyes were used \& even the mordant is also natural. Everything was collected from nature \& our surrounded area and the full dyeing process was completed by using this natural (chemical free) items.

\section{Harmful effects of chemicals used in textiles \& discus- sion about fully natural dyeing}

Mills discharge millions of gallons of this effluent as hazardous toxic waste, full of color and organic chemicals from dyeing and finishing. Presence of sulphur, naphthol, vat dyes, nitrates, acetic acid, soaps, chromium compounds and heavy metals like copper, arsenic, lead, cadmium, mercury, nickel, and cobalt and certain auxiliary chemicals all collectively make the effluent highly toxic. ${ }^{4}$ Other harmful chemicals present in the water may be formaldehyde based dye fixing agents, hydro carbon based softeners and non-bio degradable dyeing chemicals. The mill effluent is also often of a high temperature and $\mathrm{pH}$, both of which are extremely damaging. The amount of BOD, COD is more in effluent water which comes from different processes with chemicals. ${ }^{5}$ The colloidal matter present along with colors gives the water a bad appearance and foul smell. It prevents the penetration of sunlight necessary for the process of photosynthesis. This interferes with the Oxygen transfer mechanism at air water interface. ${ }^{5}$ Depletion of dissolved Oxygen in water is the most serious effect of textile waste as dissolved oxygen is very essential for marine life. In addition when this effluent is allowed to flow in the fields it clogs the pores of the soil resulting in loss of soil productivity. The texture of soil gets hardened and penetration of roots is prevented. Impurities in water affect the textile processing in many ways. In dyeing stage metallic ions present in water sometimes combine with the dyes causing dullness in shades. ${ }^{4}$ Textile effluent is a cause of significant amount of environmental degradation and human illnesses. All the organic materials present in the wastewater from a textile industry are of great concern in water treatment because they react with many disinfectants especially chlorine. Chemicals evaporate into the air we breathe or are absorbed through our skin and show up as allergic reactions and may cause harm to children even before birth.

Dyes are used for coloring the fabrics. Dyes are molecules which absorb and reflect light at specific wavelengths to give human eyes the sense of color. Dyeing in textiles is a process in which color is transferred to a finished textile or textile material (like fibers and yarns) to add permanent and long-lasting color. It can be done by hand or by machine. Dyes can come as powders, crystals, pastes or liquid dispersions, and they dissolve completely in an aqueous solution like water. ${ }^{6}$ When the textile and the dye come into contact, the textile is completely saturated by the dye and colored. ${ }^{7}$ Some of the synthetic dyes contain metals too. The natural dyes are extracted from natural substances such as plants, animals, or minerals. ${ }^{1}$ Natural dyes come from sources like plants, minerals and animals. If the dyeing procedure with natural dyes is considered, different chemical mordants are used. ${ }^{3}$ So, in dyeing there should be the presence of chemical \& dyes 
whether it is synthetic or natural. ${ }^{8}$ Fully natural dyeing means where no chemical is used starting from the initiation stage of dyeing to termination. ${ }^{9}$ In this article, dyeing is carried out by using natural dyes $\&$ natural catalyst with the use of no machine.

\section{Natural catalyst used in fully natural dyeing}

In chemical free dyeing natural catalyst was used instead of any chemical. Here the catalyst used in this chemicals free dyeing was collected only from nature. The dyes used in this process are natural dyes. The natural dye which was used is turmeric. Tamarind leaves were used as Natural Catalyst in this dyeing. The list of substance is given in Table 1.

Table I List of natural catalyst

\begin{tabular}{ll}
\hline Name & Natural catalyst \\
\hline Dyes & Turmeric \\
Natural Mordant & Tamarind Leaves \\
Chemical Mordant & $\mathrm{FeSO}_{4}$ \\
\hline
\end{tabular}

\section{Chemical structure of the substance}

The Chemical structure of the substances used in this dyeing is given below:

Turmeric can be in enol form or keto form. But the enol form is more energetically stable in the solid phase \& also in solution (Figures $1-3)$.

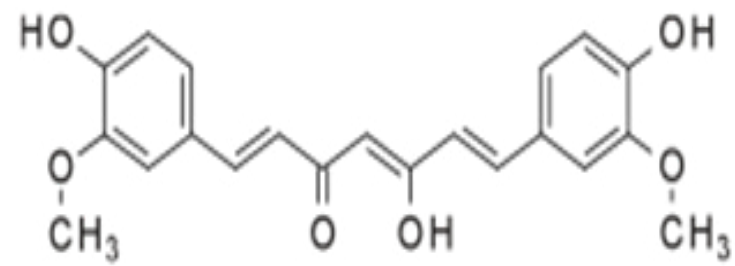

Figure I Structure of Turmeric in enol form.

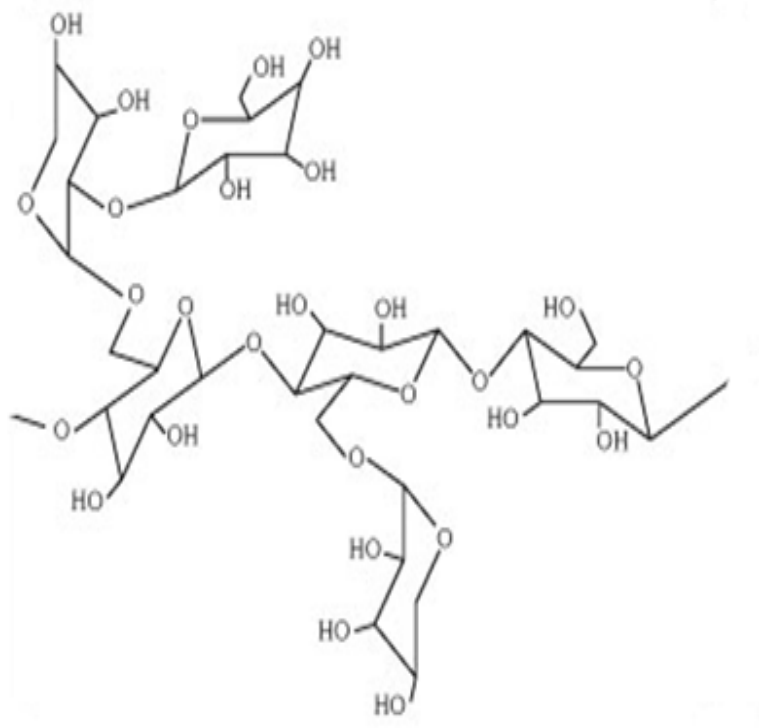

Figure 2 Structure of Tamarind which contains hydroxyl group.

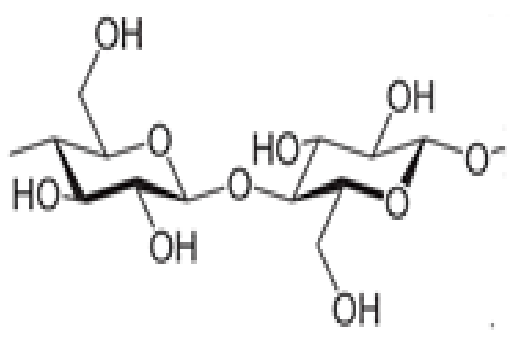

Figure 3 Structure of Cellulose.

\section{Procedure of fully natural dyeing}

At first, $2 \mathrm{~T}$ shirts (Bleached) were collected which will be dyed. After that the T-shirts were immersed into water in a pot and kept it for one hour to increase the absorbency level of the fabric to a satisfactory limit. The natural dyes from turmeric were extracted. Also collected the sap from Tamarind leaves.

Trial sample 1- First T- shirt was dyed with the natural dyes using only the natural mordant Tamarind sap (Figure 4).

Trial sample 2 which was Second T-shirt dyed with the Chemical mordant $\mathrm{FeSO}_{4}$ (Figure 5).

Before started dyeing all of the liquids were filtered. The dyeing was carried out with the mixing of natural dyes \& mordant at elevated temperature. After that the dye pot was kept to cool down. The T-shirt was then taken out from the pot and washed with normal water. After the completion of dyeing, the dyed T-shirts were washed with $3 \mathrm{~g} / 1$ detergent to make the water clean come out from the dyed product.

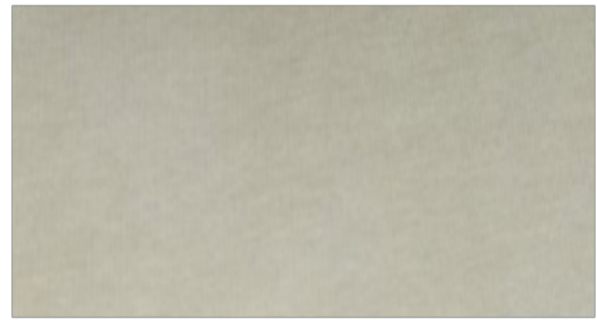

Figure 4 Trial sample I under D65 light source.

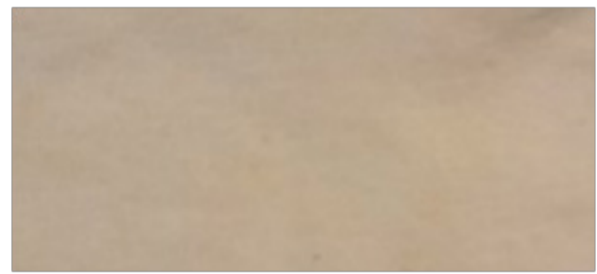

Figure 5 Trial sample 2 under D65 light source.

\section{Findings}

The dyed samples were dried. After that the quality of dyeing was checked.

a. Quality of Levelness: the levelness of the dyed samples is really good.

b. Crease mark: the fabric quality of the T-shirt was single jersey but there is no crease mark after dyeing.

c. Dye Spot: no dye spot was noticeable. 
d. Shade Variation: NO shade variation from the front and back part of the garments was found.

Here the shade of the two dyed sample is not the same as our target is to illustrate that Tamarind leaves can be used as mordant for fully natural dyeing $\&$ is good in performance point of view.

\section{Fastness observation}

The dry rubbing fastness of all two dyed sample was good 5 (Figure 6) (Figure 7).

The wet rubbing fastness of trial dyed sample 1 which was dyed only using tamarind is 5 (Figure 8).

The wet rubbing fastness of trial dyed sample 2 which was dyed only using $\mathrm{FeSO}_{4}$ is 4-5 (Figure 9).

The color fastness to wash of trial dyed sample 2 was not fully satisfactory but on the other hand the color fastness of the trial dyed sample 1 (dyed with Tamarind) was fully satisfactory and the below result illustrates that. In every composition, the rating is 4-5 (Figure 10) (Figure 11).

Note- All the fastness testing was done in Archroma Bangladesh Limited.

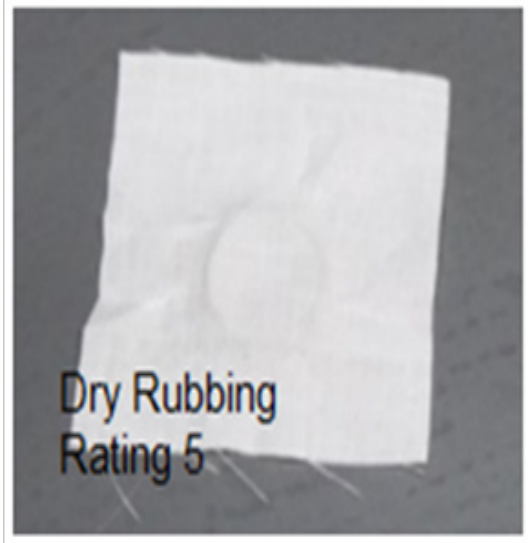

Rubbing Fastness Test//ISO $105 \times \times 12$

Figure 6 Dry Rubbing of Trial sample I.

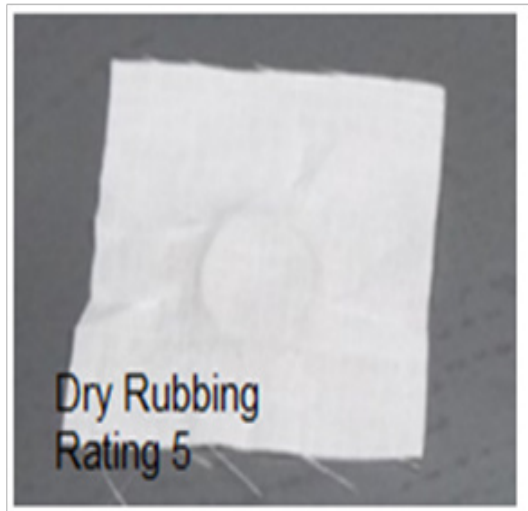

Rubbing Fastness Test//ISO $105 \cdot X 12$

Figure 7 Dry Rubbing of Trial sample 2.

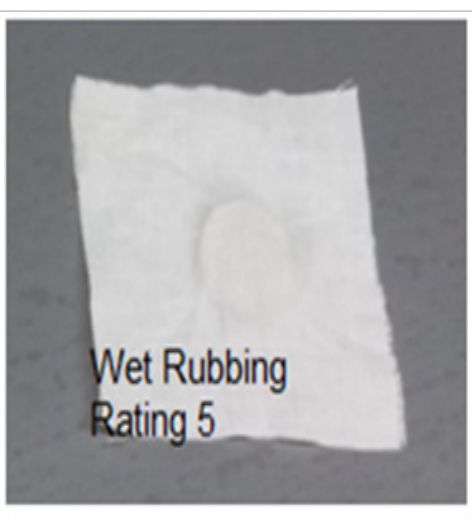

Rubbing Fastness Test//ISO $105 \cdot \times 12$

Figure 8 Wet Rubbing of Trial samplel (Rating 5).

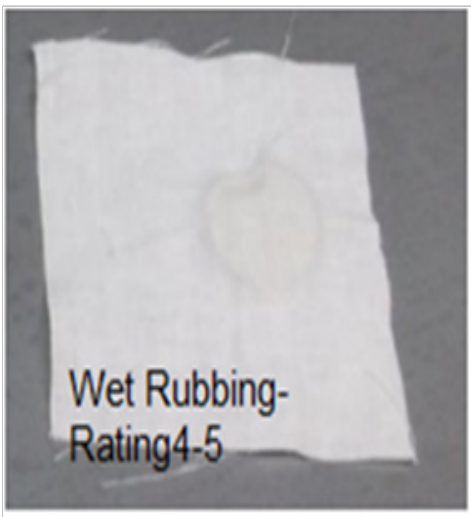

Rubbing Fastness Test//SO $105 \cdot \times 12$

Figure 9 Wet Rubbing of Trial sample I (Rating 4-5).

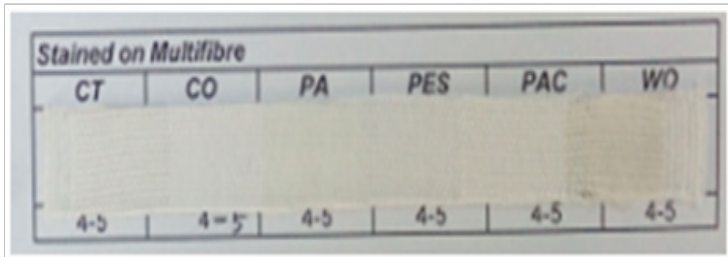

Washing Fastness Test/ISO 105 CO6 C2S

Figure 10 Color fastness to wash of trial dyed sample I (dyed with Tamarind as mordant).

\begin{tabular}{|c|c|c|c|c|c|}
\hline \multicolumn{6}{|c|}{ Stained on Multifibre } \\
\hline $\mathrm{CT}$ & $\mathrm{CO}$ & $P A$ & PES & PAC & wo \\
\hline 4.5 & 4.5 & 2.3 & $3-4$ & 4.5 & 4.5 \\
\hline
\end{tabular}

Wash Fastness to Test / ISO 105 CO6 C2S

Figure II Color fastness to wash of trial dyed sample 2 (dyed with FeSO4 as mordant). 


\section{Chemical reaction}

Tamarind leaves contain -OH group..$^{10}$ When the T shirt of cotton fabric is dyed with Turmeric, Tamarind interacts with Cellulose as shown in Figure 12.

In this chemical reaction, the Tamarind only interacts with the C-2 of cellulose unit. In reaction, the interaction happens with C-2 of cellulose. Another cellulose unit will join with C-4 by beta linkge. So, not possible to interact with $\mathrm{C}-4$, this is not possible with $\mathrm{C}-3$ due to stearis repulsion.

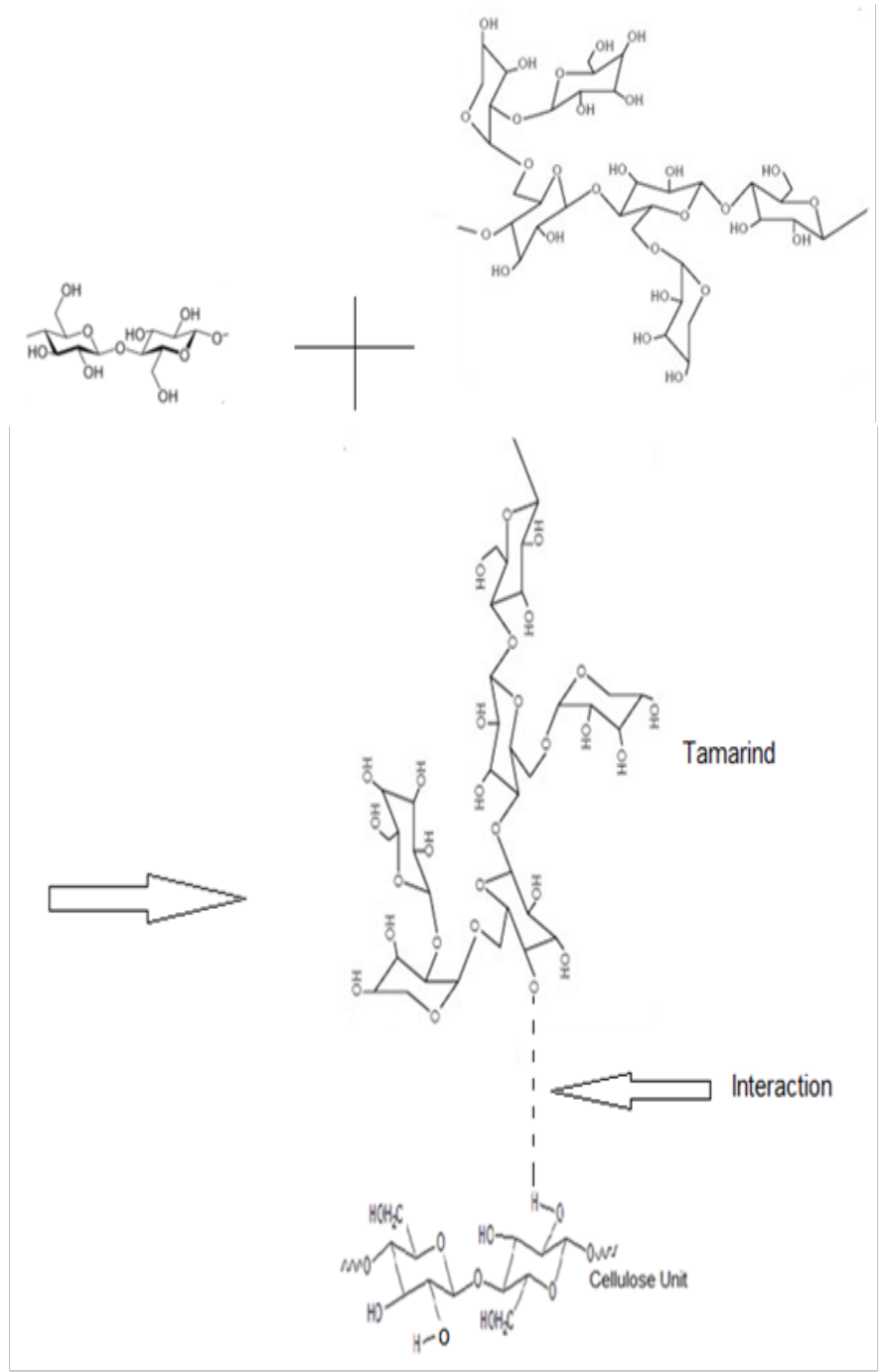

Figure 12 Cellulose interaction with Tamarind.

\section{Results}

Tamarind was used here a natural mordant. If this mordant is compared with the chemical mornadant like $\mathrm{FeSO}_{4}$, the below decisions can be reached
A. Dyeing quality performance of Tamarind is good in comparison to chemical Mordant

B. Rubbing Fastness of Tamarind is better than chemical Mordant

C. Wash Fastness of Tamarind is better than chemical Mordant

D. Tamarind is a great natural catalyst \& somehow its performance is better than chemical mordant. So, this natural mordant Tamarind can be used for ecofriendly \& fully chemical free dyeing.

\section{Conclusion}

Chemical free dyeing which is without any use of chemical is a new concept in textile dyeing. The result of this study has revealed that fully natural dyeing is possible \& the natural mordant Tamarind is a good catalyst for natural dyeing. In addition, the result of this experiment will add a bust to environmentally conscious consumers with growing need for organic clothing. This is just the step to dye naturally using Tamarind leaves as mordant but need more research to make this more convenient.

\section{Acknowledgements}

None.

\section{Conflict of interest}

Author declares there is no conflict of interest in publishing the article.

\section{References}

1. Binitha kali, Gogai N. Cationic fixing agent. Indian Textile J. 1998;108(9-12):42-46.

2. Paul R, Jayes MV, Nayak SR. Natural dyes Classification, extraction and fastness properties. Textile Dyer \& Printer. 1996;29(22):16-24.

3. Mairal AK, Shah JC. Role of some Eco-friendly chemicals in pre- and after treatments of textiles. Manmade Textile in India. 2001;(4):136-138.

4. Devi S, Katyayini VKLT, Sumanthi BS. A fast natural colour for cotton: Amaltas. New Cloth Market. 2002;16(5):13-16.

5. Devi S, Katyayini VKLT, Sumanthi BS. Optimisation of dyeing procedures. All India Co-ordinate Research Project Clothing and Textile Report. Acharya NG Ranga Agricultural University, Hyderabad; 2002. p. $20-40$.

6. International Scholarly and Scientific Research \& Innovation.

7. Agarwal A, Goel A, Paus S. Effect of mordants on natural dye. Indian Textile J. 1993;103(4):110-111.

8. Eicher JB. Nigeria Hand Crafted Textiles. University of Ife Press, Ile Ife Nigeria, Nigeria; 1976. p. 95-103.

9. Book of textile testing. Indian Standard Institute (BIS), Nanak Bhavan, New Delhi; 1982.

10. http://www.nig-magdeburg.de 